$$
p(u-v) \leqq \delta^{p}+\gamma[\eta(C)]+\int_{c(x)}^{c} \eta(s) d s .
$$

The proof follows by setting $\mu^{\prime}(s)=\eta(s), y=m-\mu[c(x)]$, where $m$ is a constant so chosen that the function $p(u-v)-y$ does not assume a positive maximum on $\partial B$.

\title{
BIBLIOGRAPHY
}

1. L. Nirenberg, A strong maximum principle for parabolic equations, Comm. Pure Appl. Math. 6 (1953), 167-177.

2. R. M. Redheffer, Bemerkungen über Monotonie und Fehlerabschätzung bei nichtlinearen partiellen Differentialgleichungen, Arch. Rational Mech. Anal. 10 (1962), 427-457.

3. S. Simoda, and M. Nagumo, Sur la solution bornếe de l'équation aux dérivées partielles du type elliptique, Proc. Japan Acad. 27 (1951), 334-339.

UNIVERSITY OF HAMBURG

\section{COMPLETE LOCALLY AFFINE SPACES AND ALGEBRAIC HULLS OF MATRIX GROUPS}

\author{
BY LOUIS AUSLANDER ${ }^{1}$ \\ Communicated by I. M. Singer, November 27, 1962
}

Let $M$ be a complete Riemann manifold with curvature and torsion zero. If $\pi_{1}(M)$ denotes the fundamental group of $M$, then Bieberbach $[3 ; 4]$ proved that $\pi_{1}(M)$ contains an abelian normal subgroup of finite index. Moreover, if $M$ is compact then $M$ is covered by a torus.

In recent years the study of general affine connections has led to the study of the following problem: How can one classify the manifolds which possess a complete affine connection with curvature and torsian zero? Such manifolds will be called complete locally affine spaces.

It was Zassenhaus [6] who first gave a general setting to the Bieberbach theorem. He showed a special case of the following theorem:

THEOREM 1. Let $G$ be a connected Lie group with its radical $R$ simply connected, $\rho: G \rightarrow G / R$ the projection, and $L$ a closed subgroup of $G$. If the identity component $L_{0}$ of $L$ is solvable, then the identity component of the closure of $\pi_{1}(L)$ is solvable.

This theorem in this generality is due to H. C. Wang [5] and his

\footnotetext{
${ }^{1}$ With partial support from the N.S. F.
} 
proof is a modification of a proof given for this theorem by the author [1] when $L$ was required to be discrete.

Now Bieberbach's theorem follows trivially from Theorem 1. However, Theorem 1 is still not strong enough to treat the general case of complete locally affine manifolds. In order to treat this general problem, we will need the following theorem.

THEOREM 2. Let $M$ be a complete locally affine manifold with fundamental group $\pi_{1}(M)$. A ssume further that the holonomy group is discrete and isomorphic to $\pi_{1}(M)$. Then $\pi_{1}(M)$ is abelian.

Using the above two theorems one can show the following:

THEOREM 3. Let $M$ be a complete locally affine manifold with fundamental group $\pi_{1}(M)$. Then $\pi_{1}(M)$ contains a normal solvable subgroup of finite index. Further if $\pi_{1}(M)$ is compact, $M$ is finitely covered by a compact solvmanifold.

COROLlaRy. Every complete locally affine manifold (compact or not) has Euler characteristic zero.

AdDEd IN PRoOF

TheOREM 4. Let $M_{i}, i=1,2$, be a compact complete locally affine manifold with fundamental group $\pi_{1}\left(M_{i}\right)$. Assume $G_{i}$ is the algebraic hull of $\pi_{1}\left(M_{i}\right)$ in the group of affine transformations. Then if $\sigma$ is an isomorphism of $\pi_{1}\left(M_{1}\right)$ onto $\pi_{1}\left(M_{2}\right)$ then $\sigma$ can be extended uniquely to an isomorphism of $G_{1}$ onto $G_{2}$.

Corollary. If $M_{1}$ and $M_{2}$ are compact complete locally affine manifolds with isomorphic fundamental groups, then $M_{1}$ is homeomorphic to $M_{2}$.

The material announced in this paper is obviously much stronger than the results already announced in [2]. A detailed account of these results will be published elsewhere.

\section{REFERENCES}

1. L. Auslander, On radicals of discrete subgroups of Lie groups (to appear).

2. - On the Euler characteristic of compact complete locally affine spaces. II, Bull. Amer. Math. Soc. 67 (1961), 405-406.

3. L. Bieberbach, Über die Bewegungsgruppen der Euklidischen Räume. I, Math. Ann. 70 (1911), 297-336.

4. - Ü̈ber die Bewegungsgruppen der Euklidischen Räume. II, Math. Ann. 72 (1912), 400-412.

5. H. C. Wang, On the deformation of lattice in a Lie group (to appear).

6. H. Zassenhaus, Beweis eines Stazes über diskrete Gruppen, Abh. Math. Sem. Univ. Hamburg. 12 (1938), 289-312.

Purdue University 